\title{
Consumo de Alcohol en Trabajadores Españoles del Sector Servicios. Variables Sociodemográficas y Laborales Implicadas
}

\section{ALCOHOL CONSUMPTION IN OCCUPATIONAL ENVIRONMENT. RELATIONSHIP WITH SOCIO-DEMOGRAPHIC AND LABOUR FACTORS}

\section{$M^{a}$ Teófila Vicente-Herrero', Ángel Arturo López-González²}

1. Servicio de Prevención de Riesgos Laborales Grupo Correos. Valencia (España). Grupo Investigación Medicina del Trabajo (GIMT). Grupo de Investigación en Salud Laboral del IUNICS (Instituto Universitario de Investigación en Ciencias de la Salud) Universitat de les Illes Balears.

2. Servicio de Prevención de Riesgos Laborales Ibsalut. Islas baleares (España). Grupo Investigación Medicina del Trabajo (GIMT). Grupo de Investigación en Salud Laboral del IUNICS (Instituto Universitario de Investigación en Ciencias de la Salud) Universitat de les Illes Balears. Profesor asociado Universidad Illes Balears. España.

\begin{abstract}
RESUMEN
Introducción. El consumo de alcohol y drogas tiene una elevada prevalencia en población general y entre trabajadores, repercutiendo en la salud laboral con incremento de enfermedades relacionadas con su consumo, accidentes laborales, absentismo, conflictividad laboral, incapacidades laborales y disminución de la productividad. Método. Estudio realizado en 7.644 trabajadores del sector servicios estableciendo relaciones entre consumo de alcohol y variables sociodemográficas: edad, sexo, nivel académico, clase social y tipo de trabajo realizado. Resultados. El mayor porcentaje de bebedores de riesgo se encuentra en la clase social más baja (65,7\% en mujeres y $96,7 \%$ en hombres), blue collar (53,7\% en mujeres y $88,5 \%$ en hombres) y no universitarios (80,6\% en mujeres y $95,9 \%$ en hombres). Son más frecuentes entre 41-50 años en las mujeres (62,7\%) y 51-60 años en los hombres (71,3\%). El consumo medio semanal en UBE (Unidades de Bebida Estándar) es superior en las mujeres y hombres de menor clase social y blue collar, y en las mujeres universitarias y hombres con estudios primarios. Conclusiones. Realizar políticas preventivas sobre consumo alcohólico en medio laboral requiere el máximo conocimiento de los aspectos con mayor repercusión en este tema, apoyo formativo y abordaje asistencial de los trabajadores afectados.
\end{abstract}

(Vicente-Herrero M, López A, 2014. Consumo de Alcohol en Trabajadores Españoles del Sector Servicios. Variables Sociodemográficas y Laborales Implicadas. Cienc Trab. Sep-Dic; 16 [51]: 158-163).

Palabras clave: CONSUMO DE ALCOHOL, SALUD LABORAL, MEDICINA DEL TRABAJO, MEDIO LABORAL.

\section{ABSTRACT}

Background: The prevalence of alcohol consumption and other drugs is high in the general population, and also among the labour population, affecting specifically the occupational health by increasing alcohol consumption-related diseases, work injuries, absenteeism, disability, work conflicts and reduced productivity.

Method: A study has been conducted with 7.644 service workers to establish the relationship between alcohol consumption and socio demographic variables such as age, sex, educational level, social class and type of work performed.

Results: The highest percentage of heavy and abusive drinkers is in the lowest social class (65,7\% in women and 96,7\% in men), blue collar (53,7\% in women and 88,5\% in men) and non-college graduates $(80,6 \%$ in women and $95,9 \%$ men). By age, this high risk drinker, are more frequent in the group 41-50 years among women $(62,7 \%)$ and $51-60$ years in men $(71,3 \%)$. The average weekly consumption in UBE is higher in women and men of lower social class, blue collar, college women and men with primary education level. Conclusions: To implement preventive policies on alcohol consumptionin the workplace is requires the maximum knowledge of the factors with the greatest impact, to enhance the implementation of prevention programs, training support, and clinical management of the affected workers.

Key Words: ALCOHOL CONSUMPTION, OCCUPATIONAL HEALTH, OCCUPATIONAL MEDICINE, OCCUPATIONAL ENVIRONMENT.

\section{INTRODUCCIÓN}

Los problemas derivados del consumo de alcohol en la población son de gran relevancia, tanto desde un punto de vista sanitario, como social o laboral. La mortalidad atribuida al consumo de

Correspondencia / Correspondence:

$\mathrm{M}^{a}$ Teófila Vicente-Herrero

Plaza del ayuntamiento $n^{\circ} 24-2$

46002 Valencia. España

Tel.: 34-963102752

Fax: 34-963940500

e-mail:mtvh@ono.com/correoteo@gmail.com

Recibido: 23 de Septiembre de 2014 / Aceptado: 03 de Octubre de 2014

alcohol en estudios llevados a cabo en la Unión Europea (UE) es un $25,5 \%$ para el hombre y un $8 \%$ para la mujer, según datos de $2004^{1}$, requiriéndose la implantación de actividades preventivas protocolizadas y coordinadas entre los distintos estamentos implicados. ${ }^{2}$ Estudios realizados en numerosos paises demuestran que el consumo de drogas y alcohol implica un incremento en el riesgo de accidentes de tráfico, algunos de los cuales ocurren en horario y lugar de trabajo ${ }^{3}$, lo que hace que este tema sea de especial interés en el ámbito laboral. La población tradicionalmente más bebedora es la masculina, tanto más en edad laboral activa, entre los 25 y los 44 años. Los estudios llevados a cabo en España reflejan que la prevalencia de consumo de alcohol en la población trabajadora supera a la de la población general ${ }^{4}$; alrededor de un $24 \%$ de los trabajadores consume una cantidad de alcohol que es considerada como de riesgo potencial para la salud física. ${ }^{5}$ 
Desde los servicios de Salud Laboral de las empresas, se atribuyen a las drogas en general y al alcohol entre ellas, efectos negativos laborales, destacando mayor absentismo, incremento de las bajas laborales, mayor accidentabilidad, disminución del rendimiento y mayor conflictividad, con un incremento de sanciones disciplinarias.

Los servicios de Salud Laboral son, junto a la Atención Primaria, el eje básico para la prevención de los problemas relacionados con el alcohol. La legislación sobre materia laboral en España parte de lo recogido en la Constitución Española de 19786, e indica el deber de los poderes públicos de velar por la seguridad e higiene en el trabajo (Art. 40), y el derecho a disfrutar de un medio ambiente laboral adecuado para el desarrollo de la persona, así como el deber de conservarlo (Art. 45). Estos deberes y derechos plantean la necesidad de un desarrollo efectivo de políticas preventivas y de promoción de la salud en el trabajo. Entre ellas se encuentran las de prevención y asistencia de problemas relacionados con el alcohol y otras drogas, que se enmarcan también en las leyes sobre Salud y Seguridad en el Trabajo Españolas ${ }^{7}$, que parten de la Directiva Marco Europea. ${ }^{8}$

Una buena parte de la vida de las personas se desarrolla en el trabajo, por lo que las condiciones laborales, sus riesgos y exigencias pueden tener una decisiva influencia en los niveles de salud y bienestar, dentro y fuera del ámbito laboral.

La gravedad de los consumos de alcohol y otras drogas en el medio laboral ha sido reconocida tradicionalmente por la Organización Internacional del Trabajo (OIT), que considera que el consumo de alcohol y de drogas es un problema que concierne a un número elevado de trabajadores. ${ }^{9}$ Señala la OIT "que los efectos nocivos del consumo de alcohol y drogas en el lugar de trabajo y en el rendimiento laboral son corrientes a todos los niveles. La seguridad profesional y la productividad pueden verse afectadas de forma adversa por empleados bajo el influjo del alcohol o las drogas. ${ }^{10}$ Indica también cómo el consumo de alcohol y drogas por los trabajadores produce un fuerte impacto, que se extiende a familia y compañeros de trabajo que son afectados en términos de pérdida de ingresos, estrés y baja moral". ${ }^{11}$ Por tanto, el consumo de alcohol -y el de otras drogas- pasa a ser un problema que va más allá del propio consumidor, para traspasar la barrera individual y adentrarse en el ambiente laboral y en la propia sociedad. En población española los datos más recientes en consumo de alcohol y perfil de consumidores lo aporta la encuesta realizada por el Ministerio de Sanidad, Servicios Sociales e Igualdad, plan nacional sobre drogas: Alcohol y drogas en población general en España 2011-2012, en una muestra de 22.128 cuestionarios válidos. Según esta encuesta, el alcohol sigue siendo la droga más consumida (76,6\% de los encuestados), si bien se observa una reducción respecto a encuestas previas, siendo mayor en hombres que en mujeres $(83,2 \%$ frente a $69,9 \%)$ y más extendido entre la población joven (entre 15-34 años). La edad media de inicio de consumo de alcohol se sitúa en los 14,7 años. En conjunto, las cifras de consumo de alcohol se muestran estabilizadas en los últimos 10 años, con un ligero descenso en las intoxicaciones etílicas en ambos sexos y en todos los grupos de edad, correspondiendo el mayor riesgo de intoxicaciones etílicas en ambos sexos al grupo de edad comprendido entre los 15-34 años. Se ha incrementado levemente la autopercepción de riesgo asociado al consumo alcohólico (por encima de 5/6 cañas o copas en fin de semana). Las medidas que la población encuestada considera como más efectivas para la actuación preventiva en consumo de alcohol son: la formación, el tratamiento, el control policial y las medidas restrictivas. Las vías de formación más solicitadas son las procedentes de medios de comunicación e Internet (redes sociales, foros, web), junto con charlas de personal sanitario especializado. ${ }^{12}$

\section{Son objetivos de este trabajo:}

Conocer los hábitos de consumo de alcohol en una muestra de población trabajadora, valorando y cuantificando el consumo referido por los trabajadores, lo que permitirá identificar como colectivos de mayor riesgo a los que tienen niveles de consumo más alto.

Aportar información de utilidad para establecer en el futuro protocolos coordinados con entidades o profesionales implicados en este tema.

Conocer la relación entre el consumo de alcohol y determinadas variables socio-demográficas y laborales.

\section{MÉTODO}

Se realiza un estudio descriptivo en 7.644 trabajadores procedentes de distintas empresas del sector servicios (administración pública) de las Islas Baleares y Comunidad Valenciana (2.828 mujeres y 4.816 hombres). Los trabajadores son elegidos aleatoriamente del total de los que acuden a los reconocimientos periódicos de vigilancia de la salud establecidos por las empresas en los años 2010 y 2011. De los 15.288 reconocimientos realizados se eligen los impares (50\% de la población).

Se solicita autorización mediante consentimiento informado a los trabajadores para la utilización de los datos obtenidos con fines epidemiológicos. Se requiere también la autorización de los Comités de Seguridad y Salud de las empresas, tal y como marca la legislación vigente en prevención de riesgos laborales española. Para valorar el consumo de alcohol durante la anamnesis se emplea un cuestionario -cumplimentado por el personal sanitario y elaborado por los autores- en el que se recoge el número de veces a la semana de consumo, el tipo de bebida y la cantidad, todo ello referido al último mes. A partir de estos datos se calculan los gramos de alcohol y las Unidades de Bebida Estándar (UBE) que en España equivalen a $10 \mathrm{~g}$ de alcohol puro. Se establece una graduación de consumo, considerando: riesgo bajo (< 14 UBE por semana en mujeres y menos de 21 en hombres), consumo de riesgo (entre 14 y 20,9 en mujeres y 21 y 34,9 en hombres) y consumo abusivo ( $>21$ en mujeres y 35 en hombres). El cálculo de las UBE se hace en base al consumo semanal y no con el mensual, siguiendo criterios estandarizados y usados como referencia por diversos autores. ${ }^{13-15}$

Se utiliza el test AUDIT-C (Alcohol Use Disorders Identification Test), de uso generalizado en consumo excesivo de alcohol y se establecen como puntos de corte para considerar bebedores de riesgo ( $\geq 4$ en mujeres $\mathrm{y} \geq 5$ en hombres).

Las variables socio demográficas y laborales utilizadas son: sexo, edad, nivel académico (elemental, secundario, universitario), clase social y tipo de trabajo.

El cálculo de clase social se realiza a partir de la clasificación nacional de ocupaciones del año 1994 (CNO-94); para ello cada uno de los puestos de trabajo de nuestro estudio es incluido en un grupo según la CNO-94. Se tiene en cuenta el listado de ocupaciones del CNO-94 a nivel del tercer dígito. Con ello los trabajadores son incluidos en uno de los 6 grupos de la clasificación 
abreviada (Grupo de Trabajo de la Sociedad Española de Epidemiología y de la Sociedad Española de Medicina Familiar y Comunitaria, 2000):

I. Directivos de la Administración Pública y de empresas de 10 o más asalariados. Profesiones asociadas a titulaciones de segundo y tercer ciclo universitario.

II. Directivos de empresas con menos de 10 asalariados. Profesiones asociadas a titulaciones de primer ciclo universitario. Técnicos, artistas y deportistas.

III. Empleados de tipo administrativo y profesionales de apoyo a la gestión administrativa y financiera. Trabajadores de los servicios personales y de seguridad. Trabajadores por cuenta propia. Supervisores de trabajadores manuales.

IVa. Trabajadores manuales cualificados.

IVb. Trabajadores manuales semicualificados.

V. Trabajadores no cualificados.

Para un mejor manejo de los grupos, a partir de estos 6, se realiza una nueva clasificación agrupando los trabajadores incluidos en I y II en la categoría de clase social 1, los trabajadores III en la categoría social 2 y finalmente los trabajadores incluidos en IVa, $\mathrm{IVb}$ y V se considerarán clase social $3 .^{16}$

La CNO-94 permite también dividir a los trabajadores en manuales (blue collar) y no manuales (white collar), para ello se tiene en cuenta el listado de ocupaciones del CNO-94 a nivel del primer dígito, del 1 al 9. Los trabajadores con un primer dígito entre $1 \mathrm{y}$

Tabla 1.

Características de la muestra según patrón de consumo y variables laborales y sociodemográficas: comparación por sexos. Porcentaje.

\begin{tabular}{|c|c|c|c|c|}
\hline & $\begin{array}{l}\text { hombres } \\
(n=4816)\end{array}$ & $\begin{array}{c}\text { mujeres } \\
(n=2828)\end{array}$ & $\chi^{2}$ & $p$ \\
\hline \multicolumn{5}{|c|}{ Patrón de consumo } \\
\hline nunca & 10,1 & 30,4 & 915,9 & $<0,0001$ \\
\hline mensual & 10,4 & 12,3 & & \\
\hline semanal & 1,2 & 0,1 & & \\
\hline fin semana & 32,7 & 40,5 & & \\
\hline $3 \mathrm{~d} /$ semana & 4,7 & 2,7 & & \\
\hline diario & 40,9 & 14 & & \\
\hline \multicolumn{5}{|l|}{ Tipo de bebida } \\
\hline vino & 29,6 & 47,6 & 226,2 & $<0,0001$ \\
\hline cerveza & 45,4 & 39,2 & & \\
\hline combinado & 24,7 & 13,2 & & \\
\hline licores & 0,3 & 0 & & \\
\hline \multicolumn{5}{|c|}{ Tipo de consumidor } \\
\hline sin riesgo & 10,1 & 30,4 & 533,4 & $<0,0001$ \\
\hline bajo riesgo & 76,7 & 60,1 & & \\
\hline de riesgo & 10,1 & 8,8 & & \\
\hline abusivo & 3,1 & 0,7 & & \\
\hline \multicolumn{5}{|l|}{ Clase social } \\
\hline clase 1 & 11 & 11,3 & 17,7 & 0,0001 \\
\hline clase 2 & 45,6 & 40,7 & & \\
\hline clase 3 & 43,4 & 47,9 & & \\
\hline \multicolumn{5}{|l|}{ Tipo de trabajo } \\
\hline white collar & 64,1 & 67,8 & 10,4 & 0,0013 \\
\hline blue collar & 35,9 & 32,2 & & \\
\hline \multicolumn{5}{|l|}{ Nivel estudios } \\
\hline primarios & 23,8 & 32,7 & 75,7 & $<0,0001$ \\
\hline secundarios & 61,5 & 52,6 & & \\
\hline universitarios & 14,6 & 14,7 & & \\
\hline \multicolumn{5}{|l|}{ Edad } \\
\hline$\leq 30 \mathrm{a}$ & 3,4 & 4,2 & 196,4 & $<0,0001$ \\
\hline $31-40 a$ & 15,9 & 13,4 & & \\
\hline $41-50 a$ & 38,4 & 49,4 & & \\
\hline $51-60 a$ & 41,1 & 29 & & \\
\hline$>60 a$ & 1,2 & 4 & & \\
\hline
\end{tabular}

4 se consideran trabajadores no manuales mientras que los trabajadores con un primer dígito comprendido entre 5 y 9 serán incluidos en la categoría de trabajadores manuales. ${ }^{17}$

Para la diferencia de proporciones se emplea la prueba de chi cuadrado; para comparar medias se emplea la t de student; y para el análisis multivariante y el cálculo de los odds ratio la regresión logística. En todos los casos, la significación estadística se establece para una $\mathrm{p}<0,05$. El estudio estadístico se realiza con el paquete G-Stat 2.0.

\section{RESULTADOS}

Las características de la muestra a estudio se presentan en la Tabla 1. Destacamos el hecho de que un $30,4 \%$ de las mujeres no consumen nunca alcohol frente a un 10\% de los hombres.

Comparando las proporciones de las diferentes variables sociodemográficas y laborales según el tipo de consumidor, en UBE por semana,

Tabla 2.

Distribución de las diferentes características laborales y sociodemográficas según nivel de consumo de alcohol en mujeres. Porcentaje.

\begin{tabular}{|c|c|c|c|c|c|}
\hline & $\begin{array}{l}\text { Consumo } \\
\text { sin riesgo* } \\
(n=860)\end{array}$ & $\begin{array}{l}\text { Consumo } \\
\text { de bajo riesgo* } \\
(n=1700)\end{array}$ & $\begin{array}{l}\text { Consumo } \\
\text { de alto riesgo } \\
(n=268)\end{array}$ & $\chi^{2}$ & $p$ \\
\hline \multicolumn{6}{|l|}{ Clase social } \\
\hline clase 1 & 9,3 & 12,2 & 11,9 & 60,7 & $<0,0001$ \\
\hline clase 2 & 39,1 & 44,5 & 22,4 & & \\
\hline clase 3 & 51,6 & 43,3 & 65,7 & & \\
\hline \multicolumn{6}{|l|}{ Tipo de trabajo } \\
\hline white collar & 60,9 & 74,6 & 46,3 & 111,3 & $<0,0001$ \\
\hline blue collar & 39,1 & 25,4 & 53,7 & & \\
\hline \multicolumn{6}{|l|}{ Nivel estudios } \\
\hline Sin estudios/primarios & 40 & 29,9 & 26,9 & 55,7 & $<0,0001$ \\
\hline secundarios & 51,6 & 52,9 & 53,7 & & \\
\hline universitarios & 8,4 & 17,2 & 19,4 & & \\
\hline \multicolumn{6}{|l|}{ Edad } \\
\hline$\leq 30$ años & 2,3 & 5,6 & 1,5 & 272,5 & $<0,0001$ \\
\hline 31-40 años & 11,6 & 15,3 & 7,5 & & \\
\hline 41-50 años & 54 & 44,9 & 62,7 & & \\
\hline $51-60$ años & 20,5 & 33,6 & 26,9 & & \\
\hline > 60 años & 11,6 & 0,5 & 1,5 & & \\
\hline
\end{tabular}

Tabla 3.

Distribución de las diferentes características laborales y sociodemográficas según nivel de consumo de alcohol en hombres. Porcentaje.

$\begin{array}{lccccc} & \begin{array}{c}\text { Sin riesgo } \\ (n=488)\end{array} & \begin{array}{c}\text { Bajo } \\ (n=3692)\end{array} & \begin{array}{c}\text { De riesgo abusivo } \\ (n=636)\end{array} & \chi^{2} & p \\ \text { Clase social } & & & & & \\ \text { clase 1 } & 13,1 & 12,4 & 1,3 & 322 & <0,0001 \\ \text { clase 2 } & 59 & 47,5 & 24,5 & & \\ \text { clase 3 } & 27,9 & 40,2 & 74,2 & & \\ \text { Tipo de trabajo } & & & & & \\ \text { white collar } & 80,3 & 67,5 & 32,1 & 357,9 & <0,0001 \\ \text { blue collar } & 19,7 & 32,5 & 67,9 & & \\ \text { Nivel estudios } & & & & & \\ \text { primarios } & 24,6 & 20,7 & 41,5 & 175,6 & <0,0001 \\ \text { secundarios } & 63,1 & 62,4 & 55,3 & & \\ \text { universitarios } & 12,3 & 16,9 & 3,1 & & \\ \text { Edad } & & & & & \\ \text { S30a } & 4,1 & 3,8 & 0,6 & 84,9 & <0,0001 \\ \text { 31-40a } & 20,5 & 16,3 & 10,7 & & \\ \text { 41-50a } & 39,3 & 39,1 & 33,3 & & \\ \text { 51-60a } & 33,6 & 39,8 & 54,7 & & \\ >\text { 60a } & 2,5 & 1,1 & 0,6 & & \end{array}$


Tabla 4.

Valores medios de consumo (UBE/semana) según caracteristicas laboralesy sociodemográficas y por sexo.

\begin{tabular}{|c|c|c|c|c|c|c|c|c|c|c|}
\hline & \multicolumn{5}{|c|}{ Mujeres } & \multicolumn{5}{|c|}{ Hombres } \\
\hline & $\mathrm{n}$ & media & $\mathrm{dt}$ & $p$ & IC 95\% & $n$ & media & $\mathrm{dt}$ & IC $95 \%$ & $p$ \\
\hline \multicolumn{11}{|l|}{ Clase social } \\
\hline clase 1 & 320 & 3,2 & 3,8 & $<0,05$ & $2,7-3,6$ & 528 & 5,6 & 5,1 & $5,2-6,1$ & $<0,05$ \\
\hline clase 2 & 1152 & 3,1 & 4,2 & & $2,9-3,3$ & 2196 & 7,6 & 8,7 & $7,3-8$ & \\
\hline clase 3 & 1356 & 4,1 & 4,7 & & $3,9-4,4$ & 2092 & 12,4 & 13,5 & $11,8-13$ & \\
\hline \multicolumn{11}{|l|}{ Tipo de trabajo } \\
\hline white collar & 1916 & 3,2 & 4,1 & $<0,05$ & $3-3,4$ & 3088 & 7,4 & 8,3 & $7,1-7,6$ & $<0,05$ \\
\hline blue collar & 912 & 4,5 & 4,9 & & $4,2-4,8$ & 1728 & 13,3 & 14 & $12,6-14$ & \\
\hline \multicolumn{11}{|l|}{ Nivel estudios } \\
\hline primarios & 924 & 3,2 & 4,4 & $<0,05$ & $2,9-3,5$ & 1148 & 12,6 & 13,7 & $11,8-13,4$ & $+<0,05$ \\
\hline secundarios & 1488 & 3,5 & 4,4 & & $3,3-3,6$ & 2964 & 9,1 & 10,6 & $8,7-9,4$ & \\
\hline universitarios & 416 & 4,6 & 4,3 & & $4,2-5$ & 704 & 6,1 & 5,9 & $5,7-6,6$ & \\
\hline \multicolumn{11}{|l|}{ Edad } \\
\hline$\leq 30 a$ & 120 & 3,7 & 2,7 & $<0,05$ & $3,2-4,1$ & 164 & 5,5 & 5 & $4,7-6,3$ & $<0,05$ \\
\hline $31-40 a$ & 380 & 2,3 & 3,1 & & $2-2,6$ & 768 & 6,9 & 8 & $6,3-7,4$ & \\
\hline $41-50 a$ & 1396 & 3,9 & 4,8 & & $3,7-4,1$ & 1848 & 9,7 & 11,1 & $9,2-10,2$ & \\
\hline $51-60 a$ & 820 & 4 & 4,5 & & $3,7-4,3$ & 1980 & 11 & 12,3 & $10,1-11,2$ & \\
\hline$>60 a$ & 112 & 0,8 & 2,8 & & $0,3-1,3$ & 56 & 10,4 & 7,7 & $8,4-12,4$ & \\
\hline
\end{tabular}

se aprecian diferencias estadisticamente significativas tanto en mujeres (Tabla 2), como en hombres (Tabla 3). En ambos sexos el mayor porcentaje de bebedores de riesgo se presenta en personas de clase social más baja (65,7\% en mujeres y 74,2\% en hombres), trabajadores manuales (53,7\% en mujeres y 67,9\% en hombres), y nivel medio de estudios (53,7\% en mujeres y 55,3\% en hombres); solo hay diferencia en lo relativo a la edad, siendo el grupo de 41 a 50 años el que más bebedores de riesgo tiene en mujeres (62,7\%) mientras en los hombres es el grupo de 51 a 60 años (54,7\%).

Cuando lo que se determina es el valor medio de consumo por semana en cada una de las variables sociodemográficas y laborales a estudio, se observa que en ambos sexos es superior en la clase social más desfavorecida (clase 3); sin embargo, en las mujeres el consumo es similar en la clase 1 y 2 mientras que en los hombres hay un incremento paulatino a medida que se desciende en la clase social. Según el tipo de trabajo, en ambos sexos, se aprecian mayores consumos en el grupo de trabajadores manuales. De acuerdo al nivel de estudios, se aprecia un comportamiento diferente según el sexo, de manera que los consumos son mayores en los niveles superiores (universitarios) en el caso de las mujeres y en los niveles inferiores (primarios) en el caso de los hombres. En las mujeres se aprecia un descenso progresivo en el consumo a medida que
Tabla 5.

Resultados de la regresión logística aplicando dos variables respuesta dicotómica.

\begin{tabular}{|c|c|c|c|c|c|}
\hline & $\beta$ & E.E. & $\mathrm{p}$ & odd ratio & IC 95\% \\
\hline \multicolumn{6}{|c|}{ Consumo-no consumo } \\
\hline edad & $-0,03$ & 0,04 & 0,3823 & 0,97 & $0,90-1,04$ \\
\hline sexo & 1,34 & 0,06 & $<0,0001$ & 3,83 & $3,38-4,33$ \\
\hline tipo trabajo & $-0,01$ & 0,1 & 0,9367 & 0,99 & $0,82-1,2$ \\
\hline clase social & 0,17 & 0,07 & 0,0099 & 1,19 & $1,04-1,36$ \\
\hline estudios & 0,35 & 0,05 & $<0,0001$ & 1,42 & $1,28-1,57$ \\
\hline \multicolumn{6}{|c|}{$\begin{array}{l}\text { Consumo de riesgo y } \\
\text { abusivo-bajo consumo }\end{array}$} \\
\hline edad & 0,13 & 0,05 & 0,0068 & 1,14 & $1,04-1,24$ \\
\hline sexo & 0,01 & 0,08 & 0,9325 & 1 & $0,86-1,18$ \\
\hline tipo trabajo & -1 & 0,11 & $<0,0001$ & 0,37 & $0,29-0,46$ \\
\hline clase social & 0,33 & 0,1 & 0,001 & 1,39 & $1,14-1,69$ \\
\hline estudios & $-0,46$ & 0,06 & $<0,0001$ & 0,63 & $0,56-0,72$ \\
\hline
\end{tabular}

$\beta=$ coeficiente beta $\quad$ E.E. $=$ error estándar de la odds ratio.

descendemos en el nivel académico, mientras que en los varones la situación es la inversa. De acuerdo a la edad, en las mujeres no se aprecia un patrón lineal de consumo, mientras que en los hombres sí se aprecia esta linealidad. En los varones el nivel medio de consumo va aumentando paralelo a la edad hasta llegar a un máximo en el grupo de 51 a 60 años. Los datos completos se presentan en la Tabla 4.

El análisis multivariante (regresión logística) para catalogar las variables sociodemográficas y laborales que más se asocian al consumo de alcohol muestra que cuando utilizamos como variable respuesta dicotómica, el consumo o no consumo, la variable que más se asocia es el sexo seguida del nivel de estudios y la clase social. Si centramos el análisis en el grupo de personas que consumen y empleamos como variable respuesta dicotómica "consumo de riesgo y abusivo" frente a "consumo bajo" permite observar que la variable que más se asocia a consumo abusivo es la clase social seguida de la edad. En este caso las odds ratio presentan valores menores a los encontrados cuando se incluían las personas no consumidoras de alcohol (Tabla 5).

El análisis de la relación entre los valores del test AUDIT-C y las variables sociodemográficas y laborales, permite apreciar cómo en las mujeres la prevalencia en los valores de AUDIT de riesgo va aumentando a medida que descendemos en la clase social (83,3\% en clase 3), nivel de estudios (72,2\% en estudios primarios) o tipo de trabajo (66,7\% en blue collar o trabajadores manuales). En los

Tabla 6.

Distribución de las características sociodemográficas y laborales según valores del AUDIT-C según el sexo del trabajador.

\begin{tabular}{|c|c|c|c|c|c|c|c|c|}
\hline & $\begin{array}{c}\text { mujeres } \\
\text { riesgo }(n=72)\end{array}$ & $\begin{array}{c}\text { hombres } \\
\text { no riesgo }(n=2756)\end{array}$ & $\chi^{2}$ & $\mathrm{p}$ & riesgo $(n=180)$ & no riesgo $(n=4636)$ & $\chi^{2}$ & $\mathrm{p}$ \\
\hline clase 1 & 0 & 11,6 & 38,2 & $<0,0001$ & 4,4 & 11,2 & 49,8 & $<0,0001$ \\
\hline clase 2 & 16,7 & 41,4 & & & 26,7 & 46,3 & & \\
\hline \multicolumn{9}{|l|}{ Tipo de trabajo } \\
\hline white collar & 33,3 & 68,7 & 40,1 & $<0,0001$ & 46,7 & 64,8 & 24,8 & $<0,0001$ \\
\hline blue collar & 66,7 & 31,3 & & & 53,3 & 35,2 & & \\
\hline secundarios & 27,8 & 53,3 & & & 48,9 & 62 & & \\
\hline universitarios & 0 & 15,1 & & & 4,4 & 15 & & \\
\hline \multicolumn{9}{|l|}{ Edad } \\
\hline$\leq 30 a$ & 0 & 4,4 & 107,2 & $<0,0001$ & 0 & 3,5 & 36,6 & $<0,0001$ \\
\hline $31-40 a$ & 0 & 13,8 & & & 2,2 & 16,5 & & \\
\hline $41-50 a$ & 16,7 & 50,2 & & & 44,4 & 38,1 & & \\
\hline $51-60 a$ & 83,3 & 27,6 & & & 51,1 & 40,7 & & \\
\hline
\end{tabular}


hombres esta pauta solo se observa en las clases sociales más bajas $(68,9 \%)$ y en los trabajadores manuales $(53,3 \%)$. En cuanto a la edad, se observa un incremento paulatino en estos mismos valores de AUDIT de riesgo en ambos sexos hasta llegar a un máximo en el grupo de 51-60 años. Los datos totales se muestran en la Tabla 6.

\section{DISCUSIÓN}

Los resultados obtenidos en este estudio muestran que el consumo de alcohol en población laboral muestra diferencias significativas según el sexo siendo superior en los varones. Igualmente se observan diferencias significativas por sexos en cuanto al tipo de consumidor, en UBE por semana, a favor también de los hombres. El consumo es superior en las clases más desfavorecidas en ambos sexos, si bien las diferencias son más marcadas en hombres que en mujeres y es mayor también en ambos sexos en el grupo blue collar. El comportamiento en consumo de alcohol y nivel de estudios es inverso en hombres y mujeres: así, en mujeres los consumos son mayores en los niveles superiores y descienden según lo hace el nivel de estudios; en el hombre ocurre al contrario y los mayores consumos son en niveles inferiores. Se puede observar que el consumo se incrementa linealmente con la edad en los varones y que las variables que más se asocian al consumo son sexo, nivel de estudios y clase social, mientras que las más asociadas al consumo de riesgo y abusivo son la clase social y la edad.

Los resultados de este trabajo tienen su comparativa en estudios previos realizados por otros autores. Nuestro trabajo se ha basado en establecer relaciones entre las distintas variables sociodemográficas y laborales con el patrón de consumo, desde un punto de vista preventivo-laboral y desde el marco de actuación del Médico del Trabajo, tratando de orientar las actuaciones preventivas que en el futuro se podrían poner en marcha, de forma que favorezca la consecución máxima de objetivos optimizando los recursos disponibles.

La revisión efectuada en la bibliografía sobre esta temática muestra que una buena parte de los estudios realizados en población laboral se han basado en cuestionarios autoadministrados, tanto de forma independiente en mujeres y hombres como de forma conjunta en ambos sexos. Así, el estudio realizado en trabajadoras chinas valorando el ambiente de trabajo como factor motivante junto con las características sociodemográficas de las trabajadoras, favorece la puesta en práctica de estudios de intervención posteriores que puedan lograr mayor efectividad considerando estas variables ${ }^{18}$, coincidiendo en este sentido con el planteamiento de nuestro trabajo.

La bibliografía muestra que el consumo abusivo de alcohol se relaciona con gran cantidad de variables, tanto laborales como extralaborales y, en ocasiones, se asocia a determinadas profesiones como en soldados o en pacientes con patologías psiquiátricas, donde es frecuente que se asocie el consumo de alcohol al de otras sustancias o drogas de abuso. Este hecho es necesario tenerlo en cuenta a la hora de establecer estrategias preventivas o de intervención precoz, siendo muy numerosos los estudios en esta línea de trabajo, donde también se relaciona con frecuencia la falta de ejercicio y la obesidad al incremento en el consumo de alcohol. ${ }^{19,20}$ En profesiones como la del médico, se ha considerado el estrés como un factor relevante en el consumo de alcohol, con mayor repercusión que el sector público que en el ejercicio privado. $^{21}$

Nuestro trabajo se ha centrado en la diferenciación de colectivos manuales y no manuales como forma de valorar la influencia del tipo de trabajo en el consumo abusivo de alcohol, especialmente si se relaciona con el nivel educacional del trabajador. Es lo que se ha venido a denominar blue and white collar. En esta línea de trabajo destaca -ya publicado en la bibliografía- el mayor consumo de alcohol en trabajadores white collar con características de alto estatus socioeconómico, jóvenes, con alto nivel de estrés en su trabajo, estilo de vida sedentario y alteraciones del sueño y del perfil lipídico. ${ }^{22}$

No cabe duda de que el conocimiento de todos los factores que pueden influir en el consumo abusivo de alcohol, tanto laborales como personales o sociales, facilita la posterior intervención, siendo en salud laboral el objetivo final la prevención precoz, especialmente si se considera la relación existente entre el consumo de alcohol y el riesgo de accidentes de trabajo asociados $y$, en especial, accidentes de tráfico en horario de trabajo. Los estudios a este respecto realizados en población general relacionan el consumo de alcohol con el incremento de accidentes, tanto cuando se considera el consumo abusivo de alcohol de forma aislada como asociado a otras sustancias. Por este motivo se recomienda una actuación conjunta de salud pública y salud laboral, puesto que solo actuando conjuntamente se podrán obtener beneficios mayores y reducir el coste en vidas humanas y el socioeconómico. . $^{23-25}$

Una muestra del interés que este tema ha despertado siempre se puede ver en la exhaustiva revisión bibliográfica realizada entre los años 1971 y 1996 sobre enfermedades y accidentes de trabajo, en un total de 311 artículos, de 72 revistas distintas y que pone en evidencia que el alcohol es uno de los factores más frecuentemente investigados en relación con los accidentes de trabajo. ${ }^{26}$

El consumo abusivo de alcohol es, en el momento actual, uno de los principales objetivos en salud ocupacional en todos los países y también en España ${ }^{27}$ y la línea de trabajo que se contempla en este trabajo con una muestra cuantitativamente importante de trabajadores del sector servicios y en el que se tratan de relacionar variables sociodemográficas y laborales con el consumo de alcohol, cuantificándolo y estratificándolo, puede ayudar, a través de un mejor conocimiento de factores implicados, a realizar una más eficaz estrategia preventiva.

El trabajo realizado desde la medicina de familia y el de unidades especializadas en consumos abusivos (UCA) cuenta con amplia experiencia en esta temática. Desde salud laboral se puede apoyar su labor con el valor añadido del conocimiento de datos laborales que puedan servir de apoyo, y con los medios al alcance y el trabajo realizado desde los servicios de prevención de las empresas. 
1. Shield KD, Gmel G, Kehoe-Chan T, Dawson DA, Grant BF, Rehm J. Mortality and potential years of life lost attributable to alcohol consumption by race and sex in the United States in 2005. PLoSOne 2013; 8(1):e51923.

2. Morris $M$, Johnson D, Morrison DS. Opportunities for prevention of alcoholrelated death in primaryc are: results from a population-based cross-sectional study. Alcohol. 2012;46(7):703-7.

3. Callaghan RC, Gatley JM, Veldhuizen $\mathrm{S}$, Lev-Ran $\mathrm{S}$, Mann R, Asbridge M. Alcohol- or drug- use disorders and motor vehicle accident mortality: a retrospective cohort study. Accid Anal Prev. 2013; 53:149-55.

4. Bastida N. Problemas laborales asociados al consumo de alcohol. Adicciones. 2002; 14(Supl.1): 239-251.

5. Ochoa E, Madoz, A. Consumo de alcohol y otras drogas en el medio laboral. Med Segur Trab. 2008; 54(213):25-32.

6. España. Constitución Española de 27 de diciembre de 1978. BOE [en línea] 1978; (311) del 29 de dic [citado 10 oct 2013). Disponible en: http://www.boe. es/legislacion/enlaces/documentos/ConstitucionCASTELLANO.pdf

7. España. Ley 31/1995, de 8 de noviembre, de Prevención de Riesgos Laborales. BOE [en línea] del 10 de noviembre de 1995 [citado10 oct 2013). Disponible en: http://www.boe.es/boe/dias/1995/11/10/pdfs/A32590-32611.pdf

8. CEE. DIRECTIVA 89/391/CEE del Consejo [en línea] del12 de junio de 1989 [citado 10 oct 2013]relativa a la aplicación de medidas para promover la mejora de la seguridad y de la salud de los trabajadores en el trabajo. Directiva Marco. Disponible en: http://eurlex.europa.eu/LexUriServ/LexUri Serv.do?uri=C ONSLEG:1989L0391:20081211:ES:PDF)

9. Organización Internacional del Trabajo. OIT. Proyecto de repertorio de recomendaciones prácticas sobre el tratamiento en los lugares de trabajo de los problemas que plantea el consumo de alcohol y drogas. Ginebra: OIT; 1944.

10. Organización Internacional del Trabajo. OIT. Lucha contra el abuso de alcohol y drogas en el lugar de trabajo. Ginebra: OIT; 1998.

11. Organización Internacional del Trabajo. OIT. Iniciativas en el lugar de trabajo para prevenir y reducir los problemas causados por el alcohol y otras drogas. Ginebra: OIT; 1988.

12. España. Ministerio de Sanidad, Servicios Sociales e Igualdad. Secretaría de Estado de Servicios Sociales e Igualdad. Delegación del Gobierno para el Plan Nacional sobre Drogas. Encuesta sobre alcohol y drogas en población general en España, edades 2011-2012 enero 2013 [en línea]. Madrid: MSSSI; 2013. [citado 10 oct. 2013]. Disponible en: http://www.msssi.gob.es/gabinetePrensa/ notaPrensa/pdf/Prese220113134906000.ppt\#256,1

13. Rodríguez-Martos A, Gual, A, Llopis JL. "Unidad de bebida estándar" como registro simplificado del consumo de bebidas alcohólicas y su determinación en España. MedClin (Barc). 1999; 112(12):446-50.

14. Guardia J, Jiménez-Arriero MA, Pascual F, Flórez G, Contel M. Guía Clínica de Alcoholismo. 2a ed. Valencia: Socidrogalcohol; 2008.
15. García del Castillo JA, Gázquez-Pertusa M, López-Sánchez C, García del Castillo-López A. Análisis del consumo de alcohol desde una perspectiva preventiva. Monografia sobre el alcoholismo. Socidrogalcohol [en línea] 2012:333-80 [citado 10 oct. 2013). Disponible en: http:// www.socidrogalcohol.org/index.php?option=com_docman...18 (consultado 1/05/2013).

16. Domingo-Salvany A, Bacigalupe A, Carrasco JM, Espelt A, Ferrando J, Borrell C. Proposals for social classclassification based on the Spanish National Classification of Occupations 2011 using neo-Weberian and neo-Marxistapproaches. GacSanit. 2013; 27(3):263-72.

17. Sánchez MA, Calvo Bonacho $E$, González $A$, Cabrera $M$, Sáinz JC, FernándezLabander C, Quevedo-Aguado L, Gelpi JA, Fernández A, Brotons $C$, de Teresa $E_{\text {, }}$ González P, Román J. High cardiovascular risk in Spanish workers. NutrMetabCardiovascDis. 2011;21(4):231-6.

18. Chen Y, Li X, Zhou Y, Zhang C, Wen X, Guo W. Alcohol consumption in relationto work environment and key sociodemographic characteristics among female sex workers in China. Subst Use Misuse. 2012;47(10):1086-99.

19. Bridger RS, Brasher K, Bennett A. Sustaining person-environment fit with a changing work force. Ergonomics. 2013;56(3):565-77.

20. Jaworowski S, Raveh D, Golmard JL, Gropp C, Mergui J. Alcohol use disorder in an Israeli general hospital: socio demographic, ethnic and clinical characteristics. IsrMedAssoc J. 2012;14(5):294-8.

21. Heponiemi $T$, Kouvonen A, Sinervo $T$, Elovainio M. Is the public healthcare sector a more strenuous working environment than the private sector for a physician? Scand J PublicHealth. 2013;41(1):11-7.

22. Tsai SY. A study of the health-related quality of life and work-related stress of white-collar migrant workers. Int J Environ Res PublicHealth. 2012; 9(10):3740-54.

23. Brady JE, Baker SP, Dimaggio C, McCarthy ML, Rebok GW, Li G. Effectiveness of mandatory alcohol testing programs in reducing alcohol involvement in fatal motor carrier crashes. Am J Epidemiol. 2009; 170(6):775-82.

24. Callaghan RC, Gatley JM, Veldhuizen S, Lev-Ran S, Mann R, Asbridge M. Alcohol- ordrug-use disorders and motor vehicle accident mortality: a retrospective cohort study. Accid Anal Prev. 2013; 53:149-55.

25. Thygerson SM, Merrill RM, Cook $\amalg$, Thomas AM. Comparison of factors influencing emergency department visits and hospitalization among drivers in work and non work-related motor vehicle crashes in Utah, 1999-2005. Accid Anal Prev. 2011;43(1):209-13.

26. Alba N, Abad F, García C. Frecuencia y caracteristicas de los artículos españoles sobre enfermedades y accidentes de trabajo a partir de la base de datos IME (1971-1996). ArchPrev Riesgos Labor 1999;2(1):4-10.

27. Rosso GL, Zanelli R, Bruno S, Feola M, Bobbio M. Professional driving and safety, a target for occupational medicine. ArchPrev Riesgos Labor 1999;2(1):4-10. 